\title{
Miranda
}

Revue pluridisciplinaire du monde anglophone /

Multidisciplinary peer-reviewed journal on the English-

speaking world

$17 \mid 2018$

Paysages et héritages de David Bowie

\section{David Bowie, la dernière vidéo star ?}

Jonathan Broda et Thomas Schmitt

\section{OpenEdition}

Journals

Édition électronique

URL : http://journals.openedition.org/miranda/13293

DOI : 10.4000/miranda. 13293

ISSN : 2108-6559

Éditeur

Université Toulouse - Jean Jaurès

Référence électronique

Jonathan Broda et Thomas Schmitt, « David Bowie, la dernière vidéo star ? », Miranda [En ligne], 17 |

2018, mis en ligne le 20 septembre 2018, consulté le 16 février 2021. URL : http://

journals.openedition.org/miranda/13293; DOI : https://doi.org/10.4000/miranda.13293

Ce document a été généré automatiquement le 16 février 2021.

\section{c) (i)}

Miranda is licensed under a Creative Commons Attribution-NonCommercial-NoDerivatives 4.0 International License. 


\title{
David Bowie, la dernière vidéo star ?
}

\author{
Jonathan Broda et Thomas Schmitt
}

David Bowie realized that video could become an opportunity for rock stars to put on (and remove) a dizzying variety of masks. Musicians had formerly turned to acting in feature films to express the multiplicity of beings trapped inside them, struggling to emerge, but now that same role-playing could be a vital aspect of the musicmaking process itself (Saul Austerlitz, Money For Nothing, New-York, Continuum, 2007, 27)

There are those stars who continually adopt new personas, often making radical changes in appearance and dress. David Bowie is the most well-known exponent of this strategy ; in the 1970s he adopted the personas of "Ziggy Stardust,", "Aladdin Sane," and "the Thin White Duke," and rock critics appeared to be confused by his decision in the 1980s to be content with his portrayal of an "ordinary" self, culminating in an appearance at the 1985 Live Aid concert in which theatrical characterization was completly absent (Andrew Goodwin, Dancing in the Distraction Factory, Mineapolis, University of Minnesota Press, 1992, 111)

1 En janvier 2016, David Bowie achève sa carrière avec Black Star. L'album et ses visuels apparaissent sur Internet comme une musique funéraire, un testament. Lors d'une ultime mise en scène, la carrière de Bowie s'achève dans la continuité d'une mise en image du musical. David Bowie fut tellement partie prenante de la création du média clip (music video) qu'il peut être considéré comme un de ses inventeurs. "Heroes ", «Ashes to Ashes » ou « China Girl » constituent des œuvres séminales qui ont défini et inspiré la grammaire, l'esthétique et les fonctions de la forme clip.

Pour commencer, nous pourrions imaginer Bowie comme un auditeur, un spectateur, voire un consommateur de la culture populaire de son temps. En tant que baby-boomer (né en 1947) Bowie a 6 ans quand le couronnement télévisé de la Reine Elisabeth II le 2 juin 1953 provoque une accélération de la vente des postes de TV... en France. ${ }^{1}$ Il a grandi en même temps que le média qui allait finalement devenir le plus important de la période. Ce média, la télévision, allait lui donner son premier grand succès en synchronisant sa chanson "Space Oddity" avec les images historiques des premiers pas d'un astronaute sur la lune. En propulsant la chanson au rang de bande originale de 
la mission Apollo 11, la BBC donnait au chanteur l'occasion unique d'atteindre un public extraordinairement large. Les premiers pas de Neil Armstrong devinrent aussi les premiers pas de Bowie dans la sphère médiatique. En guise de chapitre final, quelques semaines après la mort du chanteur, l'astronaute canadien Chris Hadfield interprétait «Space Oddity » depuis La Station Spatiale Internationale. Inspirée par le film de Stanley Kubrick 2001, L'Odyssée de l'espace (1968), la chanson fait maintenant partie de la bande originale de l'histoire de la conquête spatiale.

3 Le lien étroit qui unit l'œuvre de David Bowie et l'émancipation de la pop culture constituera un des fils rouges de notre étude. La capacité de cet artiste à comprendre que le message est le média l'a amené à créer une iconographie tout autant qu'une discographie. La multitude des personnages qu'il a créés a évolué de manière synchrone à l'évolution des médias.

4 Entre 1953 et 1969, entre le symbole même de la transition dans la continuité, celle de la tradition monarchique et l'image emblématique de la conquête moderne, la télévision s'est construit une première identité médiatique. De l'eurovision à la mondovision, la médiatisation globale télévisuelle planétaire était en marche. Quand David Bowie rencontre Mick Rock à l'orée des années 1970, il trouve en lui le partenaire idéal : un photographe désireux de mettre la musique en images. Ensemble, ils mettent sans doute au point l'esquisse du premier double de Bowie largement identifié par la sphère médiatique : Ziggy Stardust.

5 À la suite de ce premier acte fondateur, David Bowie constitue un kaléidoscope iconographique et donne à voir une multitude de facettes. Photos (pochettes d'albums, entre autres), films, performances scéniques, l'image de Ziggy se démultiplie. Aussi, l'ultime concert de Ziggy Stardust et des Spiders from Mars, le 3 juillet 1973, filmé par D.A. Pennebaker en 1973, est comparable à une cérémonie sacrificielle. D'autres figures suivront, d'autres personnages de scène ou d'écran, tels que ceux composés pour la captation de "Golden Years » lors de l'émission TV Soul Train en novembre 1975, ou dans le film The Man Who Fell on Earth (1972) de Nicholas Roeg, inaugurant alors une carrière cinématographique.

6 Ces grands ensembles iconographiques peuvent être vus comme des cercles se recoupant partiellement. Au sein du corpus le plus large nous n'avons retenu que quelques échantillons, une douzaine de chansons visuelles. Ces objets singuliers illustrent les transformations subies par les médias qui les diffusèrent. Depuis la télévision hertzienne jusqu'à YouTube en passant par le câble, ces clips (bandes vidéo mais aussi courts métrages musicaux, films promotionnels, œuvres d'art vidéo, voire proto-clips, démos ou promos) constituent un « zoo d'images ${ }^{2}$ » à classifier. S'intéresser à l'évolution de ces objets permet d'identifier les étapes de transition d'un média à l'autre. Aussi le cercle restreint auquel nous nous attachons est-il celui qui mènera insensiblement à l'avènement de MTV. Au-delà de la seule étude de l'œuvre de Bowie, c'est l'émergence d'une nouvelle forme médiatique que nous tenterons de détailler. Pour autant, une question demeure : qu'est-ce qu'un clip ? Ou plutôt, qu'aura été le clip, avant l'apparition de YouTube ? Un champ d'expérimentation, d'hybridation et de recomposition médiatique?

7 Nous examinerons les chansons visuelles créées au début des années 1970 par Mick Rock et David Bowie jusqu'à celles réalisées par David Bowie et David Mallet au début des années 1980. D’abord appelées promos ou démos, ces "videos» (néanmoins tournées sur film argentique) sont des illustrations de l'air du temps, du style et de la 
sensibilité de Bowie au fil de cette décennie. Dans la carrière de Bowie, cette période paraît décisive. Elle peut être vue comme une boucle, une circulation entre deux chansons qui symbolisent la naissance et la renaissance médiatique de l'artiste: le succès initial et protéiforme de Space Oddity ainsi que le succès globalisé (musical et iconographique) de «Let's Dance ». Cette trajectoire est synchrone avec la montée d'un désir de la part du public vis-à-vis des chansons visuelles (promos, démos, vidéos et clips) qui aboutit à la création d'émissions dédiées et finalement à la mise en route de chaînes consacrées à la diffusion de cette forme émergente. C'est notamment le cas de MTV lors de sa première phase de croissance entre 1981 et 1983. Avec ces nouveaux médias (les télévisions musicales) et cette nouvelle forme consacrée, une nouvelle culture finit par émerger, une culture clip qui aboutit à celle que nous côtoyons aujourd'hui : la culture YouTube, grâce à laquelle l'accès à la plupart des chansons visuelles est possible, sans parler des bootlegs, des remix et des versions réputées introuvables.

8 Notre corpus (1972-1983) peut sembler hétérogène voire hétéroclite puisqu'il rassemble des clips aux budgets très divers, aux diffusions très diverses et à l'esthétique très variée. En effet, au cours de cette période, Bowie a évolué, changé de cap, exploré différentes directions tout en restant étonnamment prolifique. Notre panorama présente les quatre chansons filmées en 1972 avec Mick Rock: "Life on Mars?", "John, I'm only dancing", la deuxième version de "Space Oddity " et «The Jean Genie ».

9 À la fin des années 1970, Bowie s'est rapproché d'un autre partenaire, David Mallet. Ensemble, ils ont créé les avatars de Bowie qui vont peupler 1980. Mallet coréalise la troisième version de «Space Oddity » ainsi que " Ashes to Ashes » et «Fashion ». Par la suite, en 1982-1983, le duo épouse, avec «Let's Dance » et "China Girl », les règles esthétiques de la culture MTV naissante qu'il contribue également à créer : - usage fréquent de l'allusion, de la citation (que l'on reconnaît et que l'on apprécie ${ }^{3}$ - recours à la fragmentation ou au plan séquence (rejet du montage classique) - utilisation d'une symbolique et d'un imaginaire souvent expressionniste, allégorique, "théâtral», souvent naïf.

10 La période 1972-1983 peut être envisagée comme celle de la construction d'un champ, celui de la production et de la diffusion d'objets que l'on finit par nommer, de manière générique, des clips. Ce terme a fait florès dans la presse française, en particulier dans le journal Libération, pour caractériser la nouvelle forme qui entre en interaction avec la publicité, le cinéma et la télévision. Partant d'un mode de financement pratiquement amateur (les premières promos ne bénéficient pas de l'investissement de la maison de disques et sont autofinancées par Bowie et Rock) pour aboutir à une chaîne de production standardisée, le clip a énormément évolué en tant que produit générateur d'une nouvelle économie. Il est possible de rapprocher cette émancipation du clip de phénomènes similaires dans l'histoire des médias de masse comme l'arrivée du parlant.

De plus, ce nouveau mode de consommation culturelle finit par engendrer de nouveaux produits, de nouvelles médiations physiques de la musique (comme le furent les disques), une fois les clips enregistrés sur des cassettes vidéo (VHS) sous forme notamment de compilations, d'anthologies ou de best of chronologiques. 


\section{Le concept de Rick Altman}

12 texte «De l'intermedialité au multimédia : cinéma, média, avènement du son ». Nous transposerons sa théorie sur l'avènement du son dans les années folles aux années 1972-1983, période de l'avènement du clip. À partir de 1927 jusqu'à 1935, le son s'est peu à peu intégré aux projections cinématographiques. À propos de cette mutation, R. Altman observe trois moments menant d'un média privé de l'enregistrement sonore à ce que nous nommons aujourd'hui le " cinéma parlant » : la citation, l'exploitation et la séparation. Dans ses dernières années d'hégémonie (de 1926 à 1928), le cinéma dit muet évoque fréquemment, ainsi que le fera le parlant dans son «enfance» (1930-1935), de très nombreuses représentations des autres médias (téléphones, phonographes, radio), comme si le cinéma avait besoin de s'appuyer sur la légitimité de ces autres médias pour acquérir un statut pleinement reconnu de média autonome. Sans parler du cas particulier du théâtre, les différents exemples cités par R. Altman, depuis le Don $J_{u a n^{4}}$ de 1926 et le célèbre The Jazz Singer ${ }^{5}$ de 1927, jusqu'au Say it With Songs (troisième «talkie » d'Al Jolson) et Radio Rhythm ${ }^{6}$ en 1929, Ramblin' Round Radio Row et Sing Bill Sing $^{7}$ en 1931, ou encore Rhapsody in Black and Blue en 1932, illustrent tous cette mécanique de l'intermédialité où, pour émerger et justifier son existence, un nouveau média se doit de citer puis d'exploiter les médias précédents et, finalement, de s'en séparer.

R. Altman démontre dans son texte qu'un nouveau média (ici, les soundies) se doit, lors d'une période de transition, de se référer aux médias existants pour se faire connaître, apprécier, accepter par le public. Ainsi, la télévision, un média alors inconnu, pouvait être présentée par la publicité comme une " radio visuelle.$^{8}$ Dans le domaine du showbusiness, la porosité entre le monde du music-hall et celui du grand écran est manifeste depuis la fin des années 1920, incarnée par Al Jolson (le chanteur de jazz en personne). À la suite d'Al Jolson, bon nombre d'artistes de la scène ont la possibilité de devenir des célébrités de l'écran.

Al Jolson, The World's Greatest Entertainer, apparaitrait alors comme un des premiers artistes d'une nouvelle ère médiatique. Il commence par brûler les planches de Broadway dans les années 1910 avant d'envahir les ondes de la radio des années 1920 et de faire chanter le cinéma des années 1930. De son vivant, Al Jolson a fait l'objet de deux biopics retraçant, et figeant, son incroyable célébrité. Al Jolson s'apprêtait à présenter sa première émission de télévision quand il s'est éteint en 1950. Cette trajectoire préfigure le phénomène de l'Elvis Presley mania, qui lui succède. Touchant alors une grande variété de médias (radio, disques, télévision et, rapidement, cinéma), l'engouement pour le King résulte d'un enchevêtrement nouveau d'interactions médiatiques. Bing Crosby illustre quant à lui la transition entre le système médiatique du jazz (Al Jolson) et celui du rock'n'roll (Elvis).

Dans les années 1960, le rock'n'roll devient bien plus qu'un genre musical, ce qui contraint les médias qui le diffusent à s'adapter au nouveau système qui s'instaure sous le nom de Pop Culture, phénomène procédant de la mondialisation du phénomène rock depuis Elvis jusqu'aux Beatles. L'avènement de cette Pop Culture s'accompagne de l'apparition de nouveaux modes et de nouvelles techniques comme le transistor, le juke-box, et les émissions télévisées spécialisées. Une toute nouvelle économie, fondée sur l'argent de poche d'une classe d'âge nouvellement identifiée, l'adolescence, voit le 
jour. Ce nouveau marché crée tout à la fois de nouvelles offres, de nouvelles demandes et de nouvelles habitudes de consommation. Ainsi s'ouvre l'ère des Beatles et des Rolling Stones, les idoles de la génération du baby-boom. Spectateurs de ces vingt années de mutation du système médiatique, nous spéculons que David Bowie fut intrigué dans les années 1950 par Elvis Presley, puis sans doute fasciné dans les années 1960 par John Lennon ou Mick Jagger (avec qui il travaillera plus tard). À la suite des trajectoires des Beatles et des Rolling Stones, il s'inscrit à son tour dans le cycle des évolutions médiatiques de son temps.

Ce que Rick Altman définit comme étant une citation (d'un média par un autre) au temps d'Al Jolson peut être comparé au contexte du début des années 1970 pour analyser la tactique médiatique de David Bowie. Ce dernier emprunte à des formes préexistantes - la séance photo («Life on Mars?»), la séance d'enregistrement ("Space Oddity »), la performance underground ("The Jean Genie »), le cinéma ou l'art vidéo («Ashes to Ashes ») - de la même manière que, comme le souligne Rick Altman dans son texte, le cinéma parlant a pu utiliser l'image et donc la présence du téléphone, $\mathrm{du}$ disque ou de la radio. Bowie a puisé dans la pop culture comme Al Jolson le fit avec la culture jazz, les deux artistes partageant la même stratégie de théatralisation du show-business contemporain. Comme Al Jolson, Bowie peut être considéré comme illustrant le passage de la scène à l'écran, même si cette scène et cet écran ne sont plus les mêmes qu'avant la guerre.

Nous tenterons, à travers l'examen du corpus, d'analyser une esthétique d'avant-garde devenue grand public. Aujourd'hui visionnés sur YouTube, tous ces objets qui furent projetés, télédiffusés, ou transmis sur une grande variété d'écrans paraissent sur le même plan. Ils sont en effet, de nos jours, cités, exploités et traités sur un pied d'égalité par internet.

\section{The Video Star}

Mick Rock et David Bowie ont créé ensemble quatre films promotionnels : «Life On Mars? », «John I'm only dancing », « Space Oddity " (deuxième version) et « The Jean Genie ». Ces productions audiovisuelles sont de bons exemples d'intermédialité : ce ne sont pas encore des vidéos (puisqu'elles ont été tournées et post-produites en pellicule), et elles ont beaucoup en commun avec l'esthétique d'une séance de photo pour la presse ou avec l'illustration d'une pochette de vinyle. Ces chansons visuelles ont été rétrospectivement présentées comme des exemples précoces du phénomène vidéo lorsque MTV - ainsi que bien d'autres télévisions musicales - décidèrent de diffuser ces productions pionnières dans le flot des vidéo-musiques des années 1980 et 1990.

Mick Rock a aidé David Bowie à se définir et à s'affirmer dans son identité distincte, audelà des citations de l'univers des Beatles de son tout premier album et de l'exploitation du Glam Rock qu'il contribue à créer. La collaboration entre Mick Rock et David Bowie a initié la mécanique d'une incessante et constante réinvention de l'image du chanteur. Après "Ziggy Stardust ", chaque nouvel album possède sa propre identité visuelle associée à un personnage déclinant une nouvelle facette de David Bowie : Alladin Sane, Halloween Jack, The Thin White Duke. David Bowie s'est réinventé tout en se répétant ; en un sens, sa posture anticipe l'ère du sampling, du réemploi et du recyclage, emblématique de la fin du $20^{e}$ siècle et des esthétiques postmodernes qui y sont dominantes. 


\section{Life on Mars ?}

Image emblématique de la pureté et de l'intensité, l'utilisation d'un cyclorama blanc est reprise à maintes occasions par la suite. Cet univers de blancheur immaculée sert d'écrin à un David Bowie mannequin de mode, défilant en costume bleu électrique, la chevelure rouge et le visage ultra-maquillé. L'esthétique de cette démo est particulièrement proche de la prise de vue photographique destinée à l'industrie de la mode. Le cube blanc, surnom de la galerie d'art, met ici en valeur l'artiste fait œuvre. Grâce à la blanche évanescence de l'arrière-plan, le spectateur, tel un collectionneur d'art, profite pleinement de l'ambiguïté appuyée du regard caméra. Les yeux et le regard de David Bowie sont parfaitement visibles. Le chanteur est autant un modèle qu'un performer. David Bowie attire le regard du spectateur par chacune de ses mimiques, comme le ferait un mime.

21 David Bowie est ici bien trop élégant pour qu'il s'agisse d'une interprétation de Ziggy Stardust. Il n'est pas encore non plus l'homme qui venait d'ailleurs, paraissant trop flamboyant pour cela. Pour finir, son interprétation ne correspond pas non plus au personnage récurrent de Major Tom (il ne porte ni combinaison ni casque d'astronaute). Dans "Life on Mars?", David Bowie compose un prototype, peut-être celui d'Alladin Sane, le personnage censé reprendre le flambeau au terme de la disparition de Ziggy Stardust. Or, cette élaboration se fait dans le contexte d'une reprise : Life on Mars ? est un titre de l'album «Hunky Dory » qui précède la période Ziggy. Loin d'être un processus linéaire, l'autocitation, l'exploitation et la séparation des facettes du média - de la figure, de la marque ? - procèdent d'une intrication complexe de logiques rétroactives. On trouve ici à l'œuvre la logique d'accumulation et de compilation des masques de Bowie.

Comparer cette chanson visuelle à une séance photo nous permet de faire l'hypothèse d'un réel désir de la part de l'artiste d'établir un lien intime avec un public en voie de fanatisation à travers la multiplicité des médias qui lui permettent de consommer son idole. Il est en effet possible de consommer du Bowie à la radio, sur disques, en concert, à la télévision et, quelque temps plus tard, au cinéma.

«Life on Mars? » est devenu un «classique », compilé en VHS dans les années 1980 puis en DVD dans les années 1990. Toutefois, depuis l'avènement de YouTube en 2005, un nouvel espace de comparaison s'est déployé. Ainsi Mick Rock a-t-il lui-même mis en ligne une nouvelle version de «Life on Mars? » en 2016. Dans ce cas précis, la chanson comme sa plus-value visuelle constituent des archives qui peuvent être remontées, remixées et réétalonnées comme l'illustre la tendance du remastering. Les morceaux et les clips qui resurgissent participent d'un magma fluide prêt à se fondre dans de nouveaux moules, de nouvelles visions, via de nouveaux médias. Ainsi, en France, «Life on Mars? » est devenue la bande originale d'une publicité dont le slogan est: «Ce que l'avenir vous promet, La Poste vous l'apporte.»

Ces considérations sont confortées par la nature initiale du projet puisque l'on a d'abord proposé à Bowie de traduire "Comme d'habitude"; écarté de l'entreprise "My Way ", Bowie prend sa revanche en composant sur le mode du pastiche sa propre déclinaison du succès planétaire. À un niveau plus formel, le lieu immaculé dans lequel David Bowie interprète sa chanson se rapproche de l'esthétique, dominante dans la sphère vidéo, de l'incrustation. Il s'agit là d'un premier pas vers l'esthétique vidéo et 
non plus du support film. Telles les photos illustrant un album vinyle, la couverture d'un magazine ou un poster destiné au fan, ces images sont destinées à l'appropriation par l'auditeur-spectateur. Ce dernier est en effet libre d'effectuer un découpage mental ou physique qui lui permettra de mieux illustrer son attachement à une star visuelle, mouvante et émouvante, à une image qu'il pourra punaiser, telle une pin-up, au mur de ses fantasmes.

\section{Space Oddity (deuxième version)} l'enregistrement du morceau, comme «Life on Mars? ? le faisait avec la séance photo. Le dispositif emprunte $-R$. Altman dirait « cite » - des contextes déjà médiatisés lors des reportages et des émissions spécialisées pour introduire son dispositif propre, celui de la mise en son in extenso de la chanson. En détournant les instruments de musique de leur fonction initiale pour en faire des éléments évocateurs de science-fiction, David Bowie et Mick Rock établissent un compromis entre le régime de la représentation et celui de la narration. Au-delà de l'interprétation, l'histoire évoquée dans les paroles est illustrée, même si cela est fait de manière assez pauvre. David Bowie est donc ici moins un modèle qu'un véritable musicien, un guitariste parfaitement aguerri aux techniques d'enregistrement. Instrument emblématique de l'esthétique du signal, l'oscilloscope occupe dans la mise en scène un rôle central d'analogon entre les vibrations acoustiques des cordes de la guitare et celles, électriques, qui permettent la diffusion et l'amplification du son télédiffusé. Les courbes sinusoïdales sont ici le symbole d'un nouvel âge médiatique qui s'avance, celui des video stars qui viendront se substituer aux voix sans visages de la radio. Une transition qui sera explicitée dans les paroles de la chanson et illustrée par le clip Video Killed The Radio Stars diffusée pour la première fois en France le 28 octobre 1979. ${ }^{9}$

\section{John, I'm Only Dancing}

Sans doute la mise en image la moins connue de la collection, «John, I'm Only Dancing» est, paradoxalement, la seule qui soit effectivement extraite de l'album «Ziggy Stardust ». À l'opposé de l'univers blanchâtre de «Life on Mars ? », les tonalités du décor et des costumes sont ici sombres, foncées, noires. Bowie et les Spiders from Mars sont en studio. Un montage alterné nous fait découvrir une performance de danse filmée à l'aide d'un filtre vert. Il s'agit en quelque sorte d'une proto-vidéomusique, d'un archéo-clip, le film participant à la fois d'une forme artistique voire «arty » tout en évoquant déjà ce que sera la forme standardisée, au montage très «cut ». «John, I'm Only Dancing » possède une esthétique assez brutale, la chanson visuelle se rapproche ici plus du happening artistique comme David Bowie a pu en voir lors de son séjour à la 
Factory d'Andy Warhol, à New York. En un sens, David Bowie cite ici l'esthétique underground de la production de l'avant-garde contemporaine, l'un des traits remarquables étant que Lindsay Kemp, maître de pantomime de Bowie, fait partie des interprètes. Après l'exploitation de l'esthétique « haute couture » de «Life on Mars? » et l'univers musical de "Space Oddity", nous découvrons une allusion à une autre facette artistique de David Bowie, celle de l'expression corporelle.

\section{The Jean Genie}

Film de montage, "The Jean Genie » annonce, par son rythme de coupe et sa nature hétérogène, une des tendances fortes de l'esthétique clip à naître. Mélangeant images de concert, mise en scène de studio et prises de vue documentaires, sur le vif, «The Jean Genie » fonctionne comme un kaléidoscope associant trois « reflets » : le réel, la représentation et l'image de la représentation. «The Jean Genie » est l'un des premiers exemples d'une des logiques du clip: il se donne en effet comme extrait d'un tout inexistant, making of d'un film imaginaire, symptôme ou simulacre d'une forme plus importante qui ne sera jamais réalisée, forme de bande-annonce d'un film qui n'existe pas. Le montage joue ici un rôle prépondérant, le rythme qu'il impose brouillant la perception et les catégories habituelles du cinéma : fiction, documentaire et cinéma expérimental.

\section{Space Oddity (1979) (Troisième Version)}

Diffusée lors de l'émission anglaise The Kenny Everett Video Show sur ITV en 1979, la troisième version de "Space Oddity" est un élément précieux pour comprendre l'évolution de la forme clip au sein des médias de la diffusion vidéo. Perdue parmi des centaines d'autres vidéos du genre sur YouTube, la version de 1979 apparaît comme le chaînon manquant dans la ligne d'évolution du média. Utilisant les mêmes décors, les mêmes costumes et les mêmes effets spéciaux que le célèbre « Ashes to Ashes " (diffusé quelques mois plus tard), cette version extrêmement rare, vraisemblablement diffusée une seule fois à l'époque, parait être une ébauche, un work in progress. Moitié art vidéo, moitié court métrage narratif, il s'agit de la troisième déclinaison de la chanson qui fit de David Bowie une célébrité. Le chanteur éprouvait-il le besoin d'enregistrer régulièrement le succès auquel il devait sa notoriété ? L'esthétique de la première partie est assez proche des expérimentations de Nam June Paik. David Bowie jette un regard inquiet à la caméra avant d'interrompre son chant et de cesser brusquement de jouer de la guitare. Brisant la convention scénique, il quitte l'espace de la représentation, révélant le hors champ du studio, et se dirige vers un autre lieu pour poursuivre sa prestation, cette fois-ci dans un décor de fiction, celui d'une cellule d'hôpital psychiatrique. Celui-ci apparaîtra clairement dans la vidéo de "Ashes to Ashes ». Cette chanson visuelle inaugure de nouveaux espaces de représentation. En plus du cyclorama de nouvelles scènes, électroniques, de nouveaux écrans, cathodiques, construisent un niveau différent de représentation où se projettent les artefacts vidéo. Le chanteur devient acteur, l'acteur devient personnage et le personnage devient sujet de la chanson que lui-même chante. Les diverses dimensions $\mathrm{du}$ narrateur sont alors projetées sur une multitude d'écrans. Une nouvelle logique prend dès lors en charge le montage, et la chanson elle-même prend le contrôle du clip. 
Cette mise à distance du spectateur, pouvant évoquer les théories sur le théâtre épique de Bertolt Brecht dans Nouvelles techniques d'art dramatique (1935), deviendra un des standards de l'esthétique du vidéo-clip.

Atelier de création visuelle, la version de 1979 de "Space Oddity" inaugure la séparation entre l'univers télévisuel conventionnel des programmes télévisés et une forme émergente d'expression polymorphe qui, bientôt, exigera son propre mode de diffusion, celui de la télévision musicale câblée, en un mot : MTV.

\section{Ashes to Ashes}

31 Quel est le premier clip de l'histoire... du clip ? Parmi les prétendants comme «Bohemian Rapsody» de Queen ou «Video Killed The Radio Stars» des Buggles, "Ashes to Ashes» de David Bowie (coréalisé avec David Mallet) occupe une place particulière. Pour la première fois, dans le flot incessant des diffusions télévisuelles, une production se distingue en révélant au grand public un arsenal d'effets (vidéo) jusqu'ici inédits. En particulier la palette de couleur, où prédominent les teintes magenta, détonne encore aujourd'hui par la radicalité de son choix. Plus discrètement, les fondus et incrustations d'images sont ici systématiquement utilisés non plus pour rendre le montage invisible mais au contraire pour le souligner. Quant à l'effet de solarisation de l'image il permet d'illustrer le sentiment d'un triste désert intérieur ${ }^{10}$.

«Ashes to Ashes » est bel et bien une œuvre d'art vidéo diffusée à la télévision. En cela, elle illustre l'idée de "séparation » énoncée par R. Altman. Apparaissant au début de l'ère de l'enregistrement VHS, cette chanson visuelle des plus inhabituelles est diffusée dans les night-clubs parisiens qui s'équipent alors de magnétoscopes. Immédiatement reconnue comme un chef-d'œuvre du genre, « Ashes to Ashes » continue à être diffusée régulièrement jusqu'en 1983 et après, concrétisant le nouveau départ de David Bowie vers la célébrité planétaire, celle qui le couronnera vidéo star, paradigme qu'il a finalement en grande partie créé lui-même.

\section{Let's Dance}

33 Jouer de la guitare au milieu de nulle part et laisser la musique raconter elle-même une histoire, telle est l'une des logiques qui structure «Let's Dance ». Ici, la chanson est devenue bande originale de la vidéo ${ }^{11}$. Le rythme de la batterie imite celui du montage, à moins que ce ne soit l'inverse. Et quand un personnage frappe un établi avec son marteau, c'est la basse que l'on entend. Les paroles et les sons commentent les images, la trame narrative connotant la musique. Le pont musical devient, en regard de la narration, un passage hypothétique, une alternative de fausse joie consumériste, une parodie et en même temps, un placement de produit. Non seulement la musique présente des images (allégories, symbole, métaphores) - comme c'était le cas dans "Ashes to Ashes "-, mais celles-ci procèdent d'un symbolisme explicite : racisme, lutte de classes, aliénation culturelle.

Pleinement consciente de sa propre logique capitaliste, la vidéo dénigre le paradis consumériste promis par la paire de chaussures rouges (double allusion, à The Wizard of $O z$ de Victor Fleming 1939 et à The Red shoes de Michael Powell et Emeric Pressburger en 1948). 

exhibant un effet typique de l'esthétique vidéo. Dans le même plan d'ouverture coexistent une incrustation, un ralenti et un agrandissement dynamique. Pris un à un, chacun de ces effets est déjà connu et utilisé dans la sphère filmique. Mais ici, l'utilisation de la régie vidéo donne aux différents éléments composant l'image un aspect aplati proche de celui d'une bande dessinée, en particulier quand apparaissent les fils barbelés qui font de la femme-oiseau une prisonnière. La composition de l'image est achevée quand un encadrement au motif évoquant la Chine fait son apparition, la femme prenant alors les traits d'un ex-voto mexicain, d'une icône. Plus tard dans le montage, la "fille chinoise » devient une superhéroïne dotée de super pouvoirs la rendant capable de se transformer en un éclair.

Cette forme d'hybridation de l'image illustre la transition entre l'intermédialité et le multimédia. Non seulement le clip est une forme nouvelle, mais il recycle des esthétiques plus anciennes en les superposant comme des calques. Grâce au montage, les clins d'œil au cinéma - image noir et blanc, ralentis - sont aussi intégrés. "China Girl » apparaît comme un archipel de références, d'allusions et de réminiscences. Ainsi la parure (ou la coiffe) somptueuse que revêt la jeune femme est-elle une évocation de l'opéra Turandot. La scène finale, quant à elle, cite explicitement Tant qu'il y aura des hommes (From Here to Eternity, Fred Zinnemann, 1953). Le clip emprunte à tout ce qu'il est alors techniquement possible de produire à la télévision en 1983. Le nouveau média n'est ici pas tant la caméra vidéo que la régie ou le mélangeur qui permet l'expression de ces nouveaux effets tout en émulant l'esthétique du cinéma muet, ainsi remise au goût du jour.

\section{L'incunable}

Love You till Tuesday est un film promotionnel en neuf parties tourné en 1969. Le moyen métrage comprend huit chansons visuelles ainsi qu'une pantomime de David Bowie que ce dernier narre en voix off. Intitulée " The Mask », l'histoire conte la trajectoire de son narrateur qui, un jour, se retrouve en possession d'un masque possédant le pouvoir surnaturel de faire rire tous ceux qui le voient. Qu'il l'enfile face à ses parents ou au pub, et tout le monde s'esclaffe. Mais un soir au London Paladium, le narrateur se trouve incapable de retirer le masque.

«Étranglé sur scène », titrent les journaux ; mais étonnamment aucune mention n'est faite d'un quelconque masque. Celui-ci n'aurait-il jamais existé ou bien nul autre que le protagoniste n'en avait-il connaissance ? Grâce à un procédé peu courant alliant mime et commentaire, la voix de David Bowie raconte ce que son corps illustre. Néanmoins, cette alliance du verbe et du voir n'est pas pléonastique : une distance se crée entre les 
deux canaux de communication, son et image apparaissant comme deux langages qui ne peuvent être parfaitement traduits. Si nous considérons le programme dans son ensemble - «Love You till Tuesday ", "Sell Me a Coat », "When I'm Five », "Rubber Band », «The Mask», «Let Me Sleep Beside You », «Ching-a-Ling», «Space Oddity " (première version) et «When I Live My Dream » -, nous pouvons dire qu'il possède la structure d'un spectacle de music-hall.

Le programme s'ouvre avec la chanson qui donne son nom au moyen métrage, aux 45 tours et à l'album. L'esthétique en jeu dans ces saynètes est assez proche des scopitones, ces chansons visuelles tournées en $16 \mathrm{~mm}$ qui pouvaient être regardées sur un appareil spécial, à la manière d'un juke-box, dans les bars français jusqu'à la fin des années 1970. Économe en moyens, candide, naïf, le système de représentation joue sur la variété des segments, leur diversité. Dans ce contexte, "Space Oddity (première version) peut être vu comme un précurseur de «Video Killed the Radio Star » qui, dix ans plus tard, met également en scène une science - fiction de pacotille.

41 "Love You till Tuesday » peut être considéré comme un contre-exemple, une tentative ratée dans un contexte anachronique. Tourné en couleur en février 1969, moins de deux ans après le passage de la BBC2 à la couleur lors de l'été 1967, le produit ne pouvait être destiné à passer dans Top of the Pops, diffusé en noir et blanc jusqu'en novembre 1969 sur la BBC1. Alors que la couleur était un phénomène nouveau à la télévision, le moyen métrage quelque peu terne de David Bowie n'était peut-être pas à la hauteur de ce que les Beatles avaient réalisé avec «Strawberry Fields Forever » ou « Penny Lane », filmés en janvier 1967.

42 À l'origine, le projet semble avoir été en partie destiné au marché allemand, plusieurs chansons de "Love You till Tuesday " ayant été enregistrées dans cette langue. Pour autant, le moyen métrage n'ayant pas trouvé de diffuseur, il fut plus ou moins oublié jusqu'en 1984, lorsque Kenneth Pitt, manager de David Bowie de 1967 jusqu'à 1970, le remit en circulation d'abord en VHS, puis en Laserdisc et enfin en DVD. Cette œuvre, sans public au moment de sa réalisation, apparait aujourd'hui comme une étrangeté dans le corpus, comparable à un incunable, une œuvre anachronique, ne s'intégrant dans aucun standard de production ou de diffusion. Rétrospectivement, « Love You till Tuesday " permet de se faire une idée assez précise de la théâtralité qui caractérise la démarche de David Bowie au début de sa carrière: celle d'un homme attiré par la multiplicité des arts scéniques, tiraillé entre les différentes façons de divertir le public en distrayant, chantant, racontant ou en jouant la comédie.

\section{Conclusion}

Formé au mime, clown triste, clown auguste, David Bowie a su emprunter et combiner toute sorte de masques et d'attitudes à l'univers du cinéma muet. En 1983, David Bowie est à la fois sur le grand écran avec Furyo et sur le petit avec "Let's Dance ». Un nouveau média, distinct du cinéma et de la télévision, vient d'apparaitre : le clip. Il en est une des icônes.

Depuis les premiers films sonores (phonoscènes) jusqu'aux vidéos destinées à YouTube en passant par les cinéphonies, les soundies et les scopitones, une très grande variété de productions audiovisuelles peuvent être affiliées au clip. Nous avons ainsi proposé l'appellation « chanson visuelle " pour désigner indifféremment ces formes transmises par une série de médias toujours en mutation. En étudiant quelques-unes des «songs in 
progress ", en particulier "Space Odity ", que David Bowie a écrites, composées, et adaptées aux différents écrans, nous avons proposé une autre histoire où images et sons s'influencent mutuellement.

Nous proposons ainsi de mettre en parallèle l'attitude de David Bowie vis-à-vis de sa propre célébrité, son recours perpétuel au masque et à la théâtralité, et l'émergence de la culture clip. Se recyclant sans cesse, David Bowie s'est tout au long de sa carrière présenté comme le remix de ses propres alias. Aujourd'hui, alors que l'internet est devenu un outil extraordinairement performant pour explorer l'archive, il semble en 2017 un labyrinthe rempli d'impasses. Depuis que l'accès direct au serveur est venu supplanter l'accès séquentiel de la bande VHS, la notion même de programme TV s'est brouillée.

En mettant sur le même plan d'accessibilité des vidéos matraquées plusieurs fois par heure à l'époque de leurs diffusions sur MTV et d'obscures prestations sous-diffusées, les plateformes de partage de vidéo ont créé l'illusion d'une histoire où la chronologie est à la merci d'un seul clic. Tout l'enjeu d'un discours contemporain sur les formes audiovisuelles repose sur notre capacité à penser les arts de l'enregistrement sonore et visuel, dans un temps plus long, celui du 20e siècle tout entier et non sur une seule décennie. Peut-être plus précisément qu'une pop star, David Bowie fut une vidéo star, la première, mais aussi la dernière ?

\section{BIBLIOGRAPHIE}

Altman, Rick. "De l'intermédialité au multimédia : cinéma, médias, avènement du son" CiNéMAS vol. 10, nº 1 (1999) : 37-53.

Altman, Rick. Silent Film Sound. New York : Columbia University Press, 2004.

Austerlitz, Saul. Money for nothing. New York : Continuum, 2007.

Daney, Serge. Mondino, l'as de la hanche in Le Salaire du zappeur. Paris P.O.L, 1993.

Di Marino, Bruno. Clip 20 anni di musica in video (1981-2001). Rome : Castelvecchi, 2001.

Goodwin, Andrew. Dancing in the distraction factory. Minneapolis : University of Minnesota, 1992. Jeanneney, Jean-Noël. L'écho du siècle, Dictionnaire historique de la radio et de la télévision en France. Hachette, Paris :Arte, La Cinquième, 1999.

Jullier, Laurent, Péquignot, Julien. Le clip. Histoire et esthétique. Paris : Arma,d-Colin, 2013.

Jullier, Laurent. L'écran post-moderne un cinéma de l'allusion et du feu d'artifice. Paris : L'Harmattan, 1997.

Keazor, Henry, Wübbena, Thorsten (eds.). Rewind, Play, Fast Forward. Bielefeld : Transcript, 2010.

Vernallis, Carol. Experiencing Music Video. New York : Columbia University Press, 2004. 


\section{NOTES}

1. Le couronnement de la reine Elisabeth II, en 1953, premier grand moment télévisuel... en France, et qui provoqua le premier boom dans la vente des récepteurs, fut vécu par ceux qui y assistèrent sur un petit écran (le leur, celui de leur voisin, d'un café ou d'un téléclub) avec la satisfaction des badauds dans la rue à Londres - accrue même par une mobilité interdite à ceuxci. Jeanneney Jean-Noël, L'écho du siècle, Dictionnaire historique de la radio et de la télévision en France, Hachette, Arte, La Cinquième, 1999, Paris, 606 p, page 56.

2. «Nouvelle espèce dans notre zoo d'images (les images, ne l'oublions jamais, sont des animalcules, des bêtes, des présences familières, des "meilleurs amis de l'homme »), le clip comprend deux ou trois sous-espèces et pas mal d'hybrides. » Daney Serge, « Mondino, l'as de la hanche » in Le Salaire du zappeur, P.O.L, Paris, page 103.

3. Jullier, Laurent. L'écran post-moderne un cinéma de l'allusion et du feu d'artifice. Paris: L'Harmattan, 1997, p.27

4. Le premier talkie produit par Warner Bros, réalisé par Alan Crosland avec John Barrymore, une des plus grandes vedettes de la période du cinéma muet.

5. Officiellement, le premier soundie produit by Warner Bos, réalisé par Alan Crosland avec Al Jolson, la plus grande vedette de music hall, en termes de vente de disques, de succès radiophoniques et au box office de Broadway.

6. Une adaptation de la célèbre émission de radio de Rudy.

7. Avec la super star de la radio : Bing Crosby.

8. «In fact, a television is in one sense just a radio with images. Indeed, the object that we know as a "television" could have been called "enhanced radio" or a "image radio" or a "screen radio" or even, simply a "radio". Not only could television have been called these things, but it was in fact called all of these thing during its formative period. " «Dans les faits, une télévision est en un sens une simple radio avec des images. En particulier, l'objet que nous connaissons sous le nom de «télévision » aurait pu être nommé « radio améliorée » ou "image radio» ou "radio écran » ou même, simplement, radio. Non seulement la télévision aurait pu être ainsi nommée mais elle le fut dans les faits durant sa période de formation. " Altman, Rick. Silent Film Sound. New York : Columbia University Press, 2004. p. 16

9. Schmitt, Thomas. " The Genealogy of The Clip Culture." In Ed Keazor, Henry; Wübbena Thorsten Rewind Play Fastforward, the past, present and future of the music video, transcript, Bielefeld : Transcript, 2010. 41-57.

10. Di Marino, Bruno. Clip 20 anni di musica in video (1981-2001). Rome: Castelvecchi, 2001. p.147.

11. Some visual settings for hook lines take the metaphorical possibilities of the text and extended them to remarkable lengths, as in David Bowie's «Let's Dance ».Vernallis, Carol. Experiencing Music Video. New York: Columbia University Press, 2004.

\section{RÉSUMÉS}

David Bowie est un personnage clef de l'histoire du clip. Pour analyser son influence et son apport, nous proposerons de rapprocher sa trajectoire et la trajectoire du clip, en tant que forme mais aussi en tant que média, d'un phénomène similaire d'apparition et de mutation médiatique : celui de l'arrivée du cinéma parlant. En nous appuyant sur les travaux de Rick Altman concernant les années folles, nous explorerons un corpus d'une dizaine de chansons visuelles en 
utilisant le vocabulaire mis au point par Rick Altman pour caractériser les étapes du passage du régime «muet» au régime "parlant». Pour parachever la revue de ses clips les plus connus, nous nous arrêterons sur un "incunable», tentative en partie avortée de mettre en image le premier album de David Bowie. En conclusion, nos hypothèses ne nécessitent-elles pas d'être relues à l'aune d'une histoire de la réception?

David Bowie is a key character in the the history, prehistory and of the theory of music videos. In order to analyse and understand his influence and input, we'll suggest to compare both Bowie's career and the evolution of the music video with a similar phenomenon of media mutation : the appearance of the talkies. Our study of a dozen visual songs relies on Rick Altman's work about the late 1920s, particularly his terminology to describes the stages in the evolution from "silent" to "talking" cinema. To conclude this overview of his most famous videos, we will present an aborted attempt to adapt David Bowie first album as a feature-length music video. Finally, our hypotheses will be considered in relation to the reception of Bowie's videos.

INDEX

Keywords : Pop culture, videoclip, videomusic, media history, talkies, soundies

Mots-clés : Pop culture, clip, vidéomusique, histoire des média, talkies, soundies

\section{AUTEURS}

\section{JONATHAN BRODA}

Historien du cinéma et directeur pédagogique

Eicar

jonathanbroda2@gmail.com

\section{THOMAS SCHMITT}

Journaliste

redouane.danseur@gmail.com 